\section{On Complexity of Market Equilibria with Maximum Social Welfare}

\author{
Xiaotie Deng ${ }^{1}$ and Li-Sha Huang ${ }^{2}$ \\ 1 Dept. of Computer Science, City Univ. of Hong \\ Kong, Hong Kong SAR, China \\ 2 State Key Laboratory of Intelligent Technology \\ and Systems, \\ Dept. of Computer Science and Technology, \\ Tsinghua Univ., Beijing, 10084, China
}

\begin{abstract}
We consider the computational complexity of the Market equilibrium problem by exploring the structural properties of the Leontief exchange economy. We prove that, for economies guaranteed to have a market equilibrium, finding one with maximum social welfare or maximum individual welfare is NP-hard. In addition, we prove that counting the number of equilibrium prices is \#P-hard.
\end{abstract}

Keywords Computational Complexity, NP-hardness, Market Equilibrium, Leontief Economy

\section{Introduction}

The mathematical proof of Arrow and Debreu on the price equilibrium of exchange markets [1] under a set of quite mild assumptions has provided economics with tools for rigorous studies of its subject matters. Scarf [15] designed a first algorithm and proved its convergence to such equilibrium prices in the limit that showed the possibility economic eqiulibrium state can indeed be evaluated. Subsequently, economists, mathematicians and operations researchers studied computational methods for equilibrium price with many approaches such as closed form formulas, numerical methods, as well as mathematical programming solutions, for different models of utility functions. In a recent work of Deng, Papadimitriou and Safra explicitly called for a algorithmic complexity study of the problem, and developed interesting complexity results and approximation algorithms for several classes of agent utility functions [7]. There has since been a surge of algorithmic study for computation of the price equilibrium problem with continuous variables, discovering and re-discovering polynomial time algorithms for interesting classes of agent utility functions $[9,13,8,12,10,16,2,3]$. However, as pointed out by Papadimitriou [14], hardness computational results are difficult for the problem because of the guaranteed existence of equilibrium price for the continuous variable cases under quite mild assumptions on utility functions. Indeed, the past known hardness results [7] for equilibrium price are all for indivisible goods.

A recent surprising result by Codenotti, Saberi, Varadarajan and Ye [4] proved that computing an market equilibrium of Leontief economy with certain properties is NP-hard, for which economy equilibrium may not exist at all. Informally, in a Leontief economy, each agent demands a bundle of goods proportional to a constant vector $a=\left(a_{1}, a_{2}, \ldots, a_{n}\right)^{T}$. It was known that polynomial-time algorithms existed to compute the market equilibria of Leontief economy in Fisher setting [5]. The recent hardness results were developed through a one-to-one correspondence between the market equilibria in a special case of Leontief economy and the Nash equilibria in bi-matrix games, initially discovered by Ye [17]. With that connection, NP-hard results in bi-matrix game [11] were carried over to the market equilibrium problem. Cases led to NPhardness include: (1) the uniqueness of market equilibria; (2) The existence of equilibrium with positive prices on a given set of goods; (3) The existence of equilibrium with at least (or at most) $k$ goods positive priced (c.f. [4]). Moreover, such results can be extended to prove the NP-hardness of deciding the existence of an equilibrium for Leontief economy (which is known not to have an equilibrium for some cases).

Our work tries to explore further the computational complexity issues of Arrow-Debreu equilibrium price, by fully exploring the structural properties of the Leontief economy, which is defined in Section 2. In Section 3, we focus on the pairing model and discuss social welfare issues of the equilibrium. We prove that it is NP-hard to compute the best market equilibrium in Leontief economies, in terms of social utility, even when we are restricted to cases where the existence of market price equilibrium is guaranteed. Obviously the restriction to pairing model has clear structural properties that allow NP-hardness results be easily proven. The same holds if more general utility function classes are considered. In 
Section 4, we prove the NP-hardness of maximizing individual utilities for the general model of Leontief economy. An especially interesting result is a \#P-hard proof of counting the number of market equilibrium price sets. Our hardness proofs are self-sustained and do not rely on the connection to the NP-hard problems in Nash equilibria.

\section{Exchange Market Equilibria and Utility Function Models}

We consider an exchange market which consists of $n$ divisible goods and $m$ trading agents. Each trader comes to the market with an initial endowment of goods, denoted by a vector $e^{i} \in \mathbb{R}_{+}^{n}$ for each trader $i$. W.l.o.g., we assume that the total amount of each good is normalized to 1, i.e., $\sum_{i=1}^{m} e_{j}^{i}=1,(1 \leq j \leq n)$.

Each trader has a utility function, representing its preference to different bundle of goods, which is denoted by a function $u_{i}(\cdot): \mathbb{R}_{+}^{n} \mapsto \mathbb{R}_{+}$.

At the market, the traders exchange their goods to maximized their utilities, according to the market price. They sell their initial endowments and buy their favorite goods. At a price $p \in \mathbb{R}_{+}^{n}$, trader $i$ solves the following optimization problem:

$$
\max u_{i}\left(x^{i}\right) \quad \text { s.t. } \quad\left\langle p, x^{i}\right\rangle \leq\left\langle p, e^{i}\right\rangle, x^{i} \in \mathbb{R}_{+}^{n}
$$

The market equilibrium is defined by the following requirement:

Definition 1. An Arrow-Debreu equilibrium in an exchange economy is a price vector $\bar{p} \in \mathbb{R}_{+}^{n}$ and distributions of goods $\left\{\bar{x}^{i} \in \mathbb{R}_{+}^{n}, i=1, . ., m\right\}$, such that for all $1 \leq i \leq n$,

$\overline{x^{i}} \in \operatorname{argmax}\left\{u_{i}\left(x^{i}\right) \mid\left\langle p, x^{i}\right\rangle \leq\left\langle p, e^{i}\right\rangle, x^{i} \geq 0\right\}$, and for all $1 \leq j \leq n$,

$$
\sum_{i=1}^{m} \bar{x}_{j}^{i} \leq 1
$$

we focus on a special type of utility functions called the Leontief utility function.

\subsection{Leontief Economy}

A Leontief utility function $u_{i}$ can be characterized by a vector $a^{i} \in \mathbb{R}_{+}^{n}$. The trader's utility on a bundle of goods $x^{i} \in \mathbb{R}_{+}^{n}$ is given by

$$
u_{i}\left(x^{i}\right)=\min _{1 \leq j \leq n}\left\{\frac{x_{j}^{i}}{a_{j}^{i}} \mid a_{j}^{i} \neq 0\right\}
$$

In other words, a trader with a Leontief utility function demands bundle of goods proportional to $a^{i}$. We can define a response function $d_{i}$ to present trader $i$ 's response with respect to a certain price $p$ :

$$
d_{i}(p)=\left\{\begin{array}{cl}
0, & \text { if }\left\langle p, a^{i}\right\rangle=\left\langle p, e^{i}\right\rangle=0 \\
+\infty, & \text { if }\left\langle p, a^{i}\right\rangle=0,\left\langle p, e^{i}\right\rangle \neq 0 \\
\frac{\left\langle p, e^{i}\right\rangle}{\left\langle p, a^{i}\right\rangle}, & \text { otherwise. }
\end{array}\right.
$$

Therefore, given a price $p$, trader $i$ 's demand to $\operatorname{good} j$ is $d_{i}(p) a_{j}^{i}$. Obviously, $u_{i}\left(d_{i}(p) a^{i}\right)=d_{i}(p)$. So $d_{i}(p)$ can also be viewed as the trader $i$ 's optimal utility under price $p$.

We adopt $(A, E)$ to denote a Lenotief economy, where $A$ is an $n \times m$ matrix whose $i$-th column presents trader $i$ 's demands, and $E$ is an $n \times m$ matrix whose $i$-th column is trader $i$ 's endowments.

With these notations, the equilibrium point in a Leontief economy is a pair of vectors $(p, w)$ satisfying that

$$
\begin{array}{ll}
w_{i}=d_{i}(p), & p \geq 0, p \neq 0 \\
A w \leq \mathbf{1}, & p^{T}(A w-\mathbf{1})=0
\end{array}
$$

\section{$3 \quad$ Hardness of Optimizing Social Welfare}

In this section, We consider a social welfare function as a sum of individual utilities, i.e., $\sum_{j=1}^{m} u_{j}$. Therefore, at an equilibrium state $(p, w)$ for a Leontief economy, the social welfare is $\sum_{j=1}^{m} w_{j}$. The best market equilibrium $\left(p^{*}, w^{*}\right)$ for a Leontief economy satisfies the following conditions:

$\sum_{j \geq 1} w_{j}^{*}=\max \left\{\sum_{j \geq 1} w_{j} \mid(p, w)\right.$ is an equilibrium. $\}$

Similarly, we can define the worst market equilibrium. We will show that it is NP-hard to find the best or worst market equilibrium in Leontief economies, even if the existence of an equilibrium is guaranteed.

\subsection{The Pairing Model}

In this section, our study focuses on a special pairing model introduced by Ye [17]. In the 
model, there are equal number of traders and goods and trader $i$ holds one unit of good $i$ initially. It has been proven to be very useful in building the connection between bi-matrix games and market equilibria [4].

In the pairing model for a Leontief economy, the response function of trader $i$ is simplified to:

$$
d_{i}(p)=\left\{\begin{array}{cl}
0, & \text { if } p_{i}=0=\left\langle p, a^{i}\right\rangle \\
+\infty, & \text { if } p_{i} \neq 0,\left\langle p, a^{i}\right\rangle=0 \\
p_{i} /\left\langle p, a^{i}\right\rangle, & \text { otherwise. }
\end{array}\right.
$$

This simplification introduces interesting structures that make it easy for complexity results to be established.

\subsection{Worst Social Welfare Equilibrium}

We formulate the worst social welfare equilibrium as the following decision problem:

$$
\begin{aligned}
& w^{T} \mathbf{1} \leq r \\
& \text { s.t. } \quad w_{i}=\bar{d}_{i}(p), \quad p \geq 0, p \neq 0 \\
& A w \leq \mathbf{1}, \quad p^{T}(A w-\mathbf{1})=0
\end{aligned}
$$

For the pairing model, $\left\langle p, e^{i}\right\rangle=p_{i}$. Therefore $p_{i}=0$ implies $w_{i}=d_{i}(p)=0$, and vice versa. Hence, the above complementary condition $p^{T}(A w-\mathbf{1})$ is equivalent to $w^{T}(A w-\mathbf{1})=0$.

Theorem 1. In a Leontief economy, it is NPhard to decide whether there exists a market equilibrium whose social welfare is less than an arbitrary number $r$, even if the existence of an equilibrium is already known.

Proof. Given a graph $G=(V, E)$, we construct a $2 n \times 2 n$ matrix

$$
A=\left(\begin{array}{cc}
H & \frac{1}{n} I_{n} \\
k I_{n} & \mathbf{0}
\end{array}\right)
$$

where $H=\left(h_{i j}\right)$ is defined by

$$
h_{i j}=\left\{\begin{array}{cl}
1+\frac{1}{n}, & \text { if } i=j \\
1, & \text { if } i \neq j \text { and }(i, j) \in E \\
0, & \text { otherwise. }
\end{array}\right.
$$

We claim that $G=(V, E)$ has a clique of size $k$ if and only if the worst social welfare of the above Leontief economy is no more than $r=\frac{k n}{k n+1}$.

Split the vector $w$ to $w=\left(u^{T}, v^{T}\right)^{T}$ and let

$$
S_{u}=\sum_{i=1}^{n} u_{i}, \quad S_{v}=\sum_{i=1}^{n} v_{i}, \quad S_{w}=S_{u}+S_{v}
$$

For sufficiency, let $(p, w)$ be an equilibrium point satisfying that

$$
\begin{array}{ll}
w_{i}=d_{i}(p), & S_{w} \leq k n /(k n+1) \\
A w \leq \mathbf{1}, & p^{T}(A w-\mathbf{1})=0
\end{array}
$$

We prove the existence of a $k$-clique in $G=(V, E)$ by the following four steps:

1. $S_{w} \geq \frac{k n-1}{k n}$ :

Since $A w \leq \mathbf{1}, u_{i} \leq \frac{1}{k}$ for all $i$. By the complementary condition, there exists $u_{i} \neq 0$. Hence,

$$
\begin{aligned}
1= & \left(1+\frac{1}{n}\right) u_{i}+\sum_{j \neq i} h_{i j} u_{j}+\frac{1}{n} v_{i} \\
& \leq \frac{1}{n} u_{i}+S_{w} \leq \frac{1}{k n}+S_{w}
\end{aligned}
$$

2. $S_{v} \leq \frac{k-1}{k n+1}$ :

By the complementary condition, $v_{i} \neq 0$ implies $u_{i}=1 / k$. Then for all $v_{i} \neq 0$ :

$1=\left(1+\frac{1}{n}\right) u_{i}+\sum_{j \neq i} h_{i j} u_{j}+\frac{1}{n} v_{i} \leq\left(1+\frac{1}{n}\right) S_{u}+\frac{1}{n} v_{i}$

Let $\mu=\#\left\{j \mid v_{j} \neq 0\right\}$, we have:

$1 \leq\left(1+\frac{1}{n}\right) S_{u}+\frac{1}{\mu n} S_{v}=\left(1+\frac{1}{n}\right) S_{w}-\left(1+\frac{1}{n}-\frac{1}{\mu n}\right) S_{v}$

which implies

$S_{v} \leq\left(1+\frac{1}{n}\right) S_{w}-1 \leq \frac{n+1}{n} \frac{k n}{k n+1}-1=\frac{k-1}{k n+1}$

3. \#\{i|$\left.u_{i}>0\right\} \geq k$ :

If $\left|\left\{i \mid u_{i}>0\right\}\right|<k$, there exists a $u_{i}$ such that

$$
\begin{aligned}
k u_{i} & \geq \frac{k}{k-1} S_{u}=\frac{k\left(S_{w}-S_{v}\right)}{k-1} \geq \frac{k}{k-1}\left(\frac{k n-1}{k n}-\frac{k-1}{k n+1}\right) \\
& =\frac{k n-1}{(k-1) n}-\frac{k}{k n+1}>1
\end{aligned}
$$

It is a contradiction to $u_{i} \leq \frac{1}{k}$.

4. $u_{i} \geq \frac{n}{k n+1}$ for all $u_{i}>0$ :

If $u_{i}>0$, the complementary condition shows that:

$$
\begin{aligned}
1 & =\left(1+\frac{1}{n}\right) u_{i}+\sum_{j \neq i} h_{i j} u_{j}+\frac{1}{n} v_{i} \\
& \leq \frac{1}{n} u_{i}+S_{w} \leq \frac{1}{n} u_{i}+\frac{k n}{k n+1}
\end{aligned}
$$

which implies

$$
u_{i} \geq n\left(1-\frac{k n}{k n+1}\right)=\frac{n}{k n+1}
$$


The last two assertions prove that there must exist exactly $k$ indices $\left\{i_{1}, i_{2}, \ldots, i_{k}\right\}$ such that they are the only non-zero entries in $w$ and $u_{i_{l}}=\frac{n}{k n+1}$ for all $1 \leq l \leq k$.

All the inequalities in $u_{i} \neq 0$ and

$$
1=\left(1+\frac{1}{n}\right) u_{i}+\sum_{j \neq i} h_{i j} u_{j}+\frac{1}{n} v_{i} \leq \frac{1}{n} u_{i}+S_{w}
$$

hold in equality. Therefore, all $g_{i j}=1$ for non-zero $u_{i}$ and $u_{j}$. Therefore, the vertices $\left\{i_{1}, i_{2}, \ldots, i_{k}\right\}$ form a $k$-clique.

The necessity is easy. If the vertices $\{1,2, \ldots, k\}$ form a $k$-clique in $G=(V, E)$, set $w_{i}=\frac{n}{k n+1}$ and $p_{i}=1$ for $i \leq k$ and $w_{j}=p_{j}=0$ for $j>k$. Then $(p, w)$ satisfies

$$
\begin{array}{ll}
w_{i}=d_{i}(p), & S_{w} \leq k n /(k n+1) \\
A w \leq \mathbf{1}, & p^{T}(A w-\mathbf{1})=0
\end{array}
$$

The economies defined above always admits an equilibrium: Let $u_{1}=1 / k, v_{1}=n-n / k-1 / k$ and any other components of $w$ be zeros. Let $p_{1}=1$ and $p_{n+1}=1-1 / k-1 / k n$. It is easy to verify that $(p, w)$ is an equilibrium.

\subsection{Best Social Welfare Equilibrium}

The above proof has prepared us with techniques for studying the more interesting maximization problem defined as follows.

$$
\begin{array}{cl}
\max \quad w^{T} \mathbf{1} & \\
\text { s.t. } \quad w_{i}=d_{i}(p), & p \geq 0, p \neq 0 \\
A w \leq \mathbf{1}, & p^{T}(A w-\mathbf{1})=0
\end{array}
$$

Theorem 2. In a Leontief economy, it is NPhard to decide whether there exists a market equilibrium whose social welfare is greater than an arbitrary number $r$, even if the existence of an equilibrium is already known.

Proof. We carry out the proof by a reduction from CLIQUE. Given a graph $G=(V, E)$ with $n$ vertices and an integer $k \leq n$, we can construct a matrix $A$ using the construction in the proof of Theorem 1. Define an $(2 n+2) \times(2 n+2)$ matrix $B$ as follows:

$$
B=\left(\begin{array}{ccc}
A & 0 & 0 \\
\mathbf{1}^{T} & 0 & \varepsilon \\
\mathbf{0}^{T} & 1 & 0.5
\end{array}\right)
$$

where $\varepsilon=\frac{1}{2(k n+1)}$.

Let $z=\left(w^{T}, x, y\right)^{T} \in \mathbb{R}^{2 n+2}$, in which $w \in$ $\mathbb{R}^{2 n}, x, y \in \mathbb{R}$. Let $S_{w}=\sum_{i=1}^{2 n} w_{i}$ and $S_{z}=S_{w}+$ $x+y$. Consider the following problem:

$$
\begin{gathered}
\max S_{z} \\
\text { s.t. } \quad z_{i}=d_{i}(p), \quad p \geq 0, p \neq 0 \\
B z \leq \mathbf{1}, \quad p^{T}(B z-\mathbf{1})=0
\end{gathered}
$$

We claim that there is a $k$-clique in $G$ if and only if there is an equilibrium $(p, z)$ such that $S_{z} \geq 2+\frac{k n}{k n+1}$.

The necessity is easy. For each node $i$ in the kclique, set $u_{i}=\frac{n}{k n+1}$ and set any other variables in $w$ zeros. Set $x=0$ and $y=2$.

For the sufficiency, assume that there exists an equilibrium $(p, z)$ such that:

$$
\begin{aligned}
z_{i}=d_{i}(p), & S_{z} \geq 2+\frac{k n}{k n+1} \\
B z \leq \mathbf{1}, & p^{T}(B z-\mathbf{1})=0
\end{aligned}
$$

If $w=0$, then $S_{z} \leq 2$ because $x+0.5 y \leq 1$, a contradiction. Therefore $w \neq 0$. Since $B z \leq \mathbf{1}$, we have $S_{w} \leq 1$ and $x \leq 1$. If $y=0, S_{z}=$ $S_{w}+x \leq 2$, a contradiction. Therefore $y>0$. By the complementary condition, $x=1-0.5 y$.

From $B z \leq \mathbf{1}$, we have $S_{w}+y \varepsilon \leq 1$. Note that $\varepsilon=\frac{1}{2(k n+1)}$, then

$$
\begin{aligned}
S_{z} & =S_{w}+x+y=S_{w}+(1-0.5 y)+y \\
& =S_{w}+1+0.5 y \leq S_{w}+1+\left(1-S_{w}\right) /(2 \varepsilon) \\
& =2+k n-k n S_{w}
\end{aligned}
$$

With our assumption that $S_{z} \geq 2+\frac{k n}{k n+1}$, it follows that $k n S_{w} \leq k n-\frac{k n}{k n+1}$. Therefore, $S_{w} \leq$ $\frac{k n}{k n+1}$.

By the assertion in the proof of Theorem 1, there exists a k-clique in $G$.

Similar to the end of Theorem 1, we can find an equilibrium for the economy defined above.

\section{Hardness of Optimizing Individual Utility}

In this section, we will show that it is NP-hard to find the equilibrium which maximize a certain trader's utility, even if the existence of an equilibrium is already known. We also prove that counting the number of equilibria is \#P-complete.

\subsection{Min-Max of Individual Utility and Count the Number of Equilibria}

Let $\phi$ be a Boolean formula in 3-conjunctive normal form. Let $V=\left\{x_{1}, x_{2}, \ldots, x_{n}\right\}$ be its set of variables, $L=\left\{l_{1}^{0}, l_{1}^{1}, l_{2}^{0}, l_{2}^{1}, \ldots, l_{n}^{0}, l_{n}^{1}\right\}$ the set of corresponding literals and $C=\left\{c_{1}, c_{2}, \ldots, c_{m}\right\}$ its set of clauses. 
We will construct a Lenotief economy $(A, E)$ for $\phi$. Let $B$ denote the set of traders and $G$ denote the set of goods.

There are $|V|+|L|+1=3 n+1$ traders in the economy, denoted by

$$
B=\left\{x_{1}, x_{2}, \ldots, x_{n}\right\} \cup\left\{l_{1}^{0}, l_{1}^{1}, l_{2}^{0}, l_{2}^{1}, \ldots, l_{n}^{0}, l_{n}^{1}\right\} \cup\{\phi\}
$$

There are $|V|+|L|+|C|=3 n+m$ goods in the economy. We also denote them by

$$
\begin{aligned}
G= & \left\{x_{1}, x_{2}, \ldots, x_{n}\right\} \cup\left\{l_{1}^{0}, l_{1}^{1}, l_{2}^{0}, l_{2}^{1}, \ldots, l_{n}^{0}, l_{n}^{1}\right\} \\
& \cup\left\{c_{1}, \ldots, c_{m}\right\}
\end{aligned}
$$

The ambiguity of using the same symbol for both the trades and the goods should be made clear from the context.

Let $A(b, g)$ denote trader $b$ 's demand for good $g$ in proportion and $E(b, g)$ denote trader $b$ 's initial endowment of good $g$. Then the economy $(A, E)$ is defined as follows:

$$
A(b, g)= \begin{cases}1, & \text { if } b=x_{i}, g=l_{i}^{j}, 1 \leq i \leq n, j=0,1 \\ 1, & \text { if } b=l_{i}^{j}, g=x_{i}, 1 \leq i \leq n, j=0,1 \\ 1, & \text { if } b=g=l_{i}^{j}, 1 \leq i \leq n, j=0,1 \\ 1, & \text { if } b=l_{i}^{j}, g=c_{k}, l_{i}^{j} \in c_{k}, \forall i, j, k \\ 2, & \text { if } b=l_{i}^{j}, g=c_{k}, l_{i}^{j} \notin c_{k} \\ & \text { and } l_{i}^{1-j} \in c_{k}, \forall i, j, k ; \\ 0, & \text { otherwise. }\end{cases}
$$

$$
E(b, g)=\left\{\begin{array}{cl}
1, & \text { if } b=g=l_{i}^{j}, \forall i, j \\
1 / n, & \text { if } b \in V, g \in V \cup L \\
5, & \text { if } b=\phi, g \in C \\
0, & \text { otherwise. }
\end{array}\right.
$$

In the economy $(A, E)$, the total amount of good $g$ is 1 (for $g \in V$ ), 2 (for $g \in L$ ) or 5 (for $g \in C)$.

Theorem 3. For a Leontief economy and an arbitrary number $r$, it is NP-hard to decide whether there exists an equilibrium in which the maximal individual utility is less than $r$.

Proof. We prove the theorem by showing that $\phi$ is satisfiable if and only if there exists an equilibrium in the corresponding Leontief economy such that the maximal individual utility in the equilibrium is no more than 1.

For the necessity, let there be a truth assignment $\left(l_{1}, \ldots, l_{n}\right)$ satisfies $\phi$. Set $P(g)=1$ for all $g=l_{i}^{j}=l_{i}$ and $P(g)=0$ otherwise. If $b=\phi$ or $b=l_{i}^{j} \neq l_{i}$, the initial endowment of $b$ would be valued as zero. By our convention of defining the response function, those agents will not want anything and their utility values will be zero.

If $b=x_{i}$, then $E(b, g)=1 / n$ for all $g \in V \cup L$. It follows that the value of initial endowment of $b$ is $\frac{1}{n} \times n=1$ as $P(g)=1$ for $g=l_{i}^{j}=l_{i}$ and $P(g)=0$ otherwise. If $b=l_{i}^{j}=l_{i}$, then the value of initial endowment of $b$ is also 1 as both $E(b, g)=1$ and $P(g)=1$ for $b=g=l_{i}^{j}=l_{i}$.

By definition of Leontief utility functions, we derive the utilities $U(b)$ of agent b as follows:

$$
U(b)=\left\{\begin{array}{l}
1, \text { when } b=x_{i}, \forall 1 \leq i \leq n \\
1, \text { when } b=l_{i}^{j}=l_{i}, \forall 1 \leq i \leq n \\
0, \text { when } b=l_{i}^{j} \neq l_{i}, \forall 1 \leq i \leq n \\
0, \text { when } b=\phi
\end{array}\right.
$$

For the Leontief economy, the trader b's demand for good $g$ is $\mathrm{A}(\mathrm{b}, \mathrm{g}) \mathrm{U}(\mathrm{b})$. Therefore, $\sum_{b} A(b, g) U(b)$ is the total amount of consumed good g. And $\sum_{g} A(b, g) P(g) U(b)$ is the total amount of money spent by trader $b$. The equilibrium conditions are:

1. $\sum_{g} A(b, g) P(g) U(b) \leq \sum_{g} E(b, g) P(g), \forall b \in B$ 2. $\sum_{b} A(b, g) U(b) \leq \sum_{b} E(b, g), \forall g \in G$ 3. $\sum_{g} P(g)\left(\sum_{b} A(b, g) U(b)-\sum_{b} E(b, g)\right)=0$

For the first set of inequalities, we only need to consider those traders with endowment of nonzero value under the price vector $P$ defined above, since other traders will demand nothing according to the definition of the Leontief utility functions.

1. For agent b: $b=x_{i}$, it desires two types of goods $l_{i}^{0}$ and $l_{i}^{1}$, of which one's price is zero and another's price is one. Therefore, with one unit of wealth, it will acquire one unit each of $l_{i}^{0}$ and $l_{i}^{1}$.

2. For agent b: $b=l_{i}^{j}=l_{i}$, it desires goods $g=x_{i}$ and $g=l_{i}^{j}, g=c_{k}$ such that $l_{i}^{j} \in c_{k}$, as well as $g=c_{k}$ such that $l_{i}^{j} \notin c_{k}$ but $l_{i}^{1-j} \in c_{k}$. All those goods cost zero except $g=l_{i}^{j}$ cost one per unit. Therefore, $b=l_{i}^{j}$ will get one unit of $g=l_{i}^{j}$, one unit of $x_{i}$, one unit of $c_{k}$ if $l_{i}^{j} \in c_{k}$, and two units of $g=c_{k}$ if $l_{i}^{j} \notin c_{k}$ but $l_{i}^{1-j} \in c_{k}$.

For the second set of inequalities, consider goods in $V \cup L$ :

$\sum_{b} A(b, g) U(b)=1=\sum_{b} E(b, g), \forall g \in V$
$\sum_{b} A(b, g) U(b)=2=\sum_{b} E(b, g)$, for $g=l_{i}^{j}=l_{i}$
$\sum_{b} A(b, g) U(b)=1<\sum_{b} E(b, g)$, for $g=l_{i}^{j} \neq l_{i}$

For goods in $C$, one unit of $g=c_{k}$ is desired by each literal in $c_{k}$ that is true, two units of $g=c_{k}$ is desired by each literal in $c_{k}$ that is 
false. Since $l_{i}$ 's, $i=1,2, \ldots, n$, is a satisfying assignment, there is a true literal in each $c_{k}$ of three literals. Therefore, at most five units of $g=c_{k}$ are desired. With 5 units of each type of such goods, we have enough for them.

Finally, as the price of $g=l_{i}^{1-j}$ with $l_{i}=l_{i}^{j}$ is zero, and the prices of all $c_{k}$ 's are all zero, we have

$$
\sum_{g} P(g)\left(\sum_{b} A(b, g) U(b)-\sum_{b} E(b, g)\right)=0
$$

For the sufficiency of the theorem, assume there is an equilibrium $(P, U)$ such that every trader's utility is no more than 1.

If $\left.P\right|_{V \cup L}=0$, the value of initial endowments of all traders in $V \cup L$ will be zero. Then the price of goods in $C$ will be zero too. It is a contradiction, so $\left.P\right|_{V \cup L} \neq 0$. Then the budget of every trader in $V$ is positive. Since any trader $x_{i} \in V$ only wants the goods $l_{i}^{0}$ and $l_{i}^{1}$. Therefore, either $P\left(l_{i}^{0}\right)$ or $P\left(l_{i}^{1}\right)$ is positive. Otherwise, $x_{i}$ would buy an infinite amount of them.

Assume $P\left(l_{i}^{j}\right)>0$, then trader $l_{i}^{j}$ has positive budget since its initial endowment is one unit of $l_{i}^{j}$. However, since $l_{i}^{j}$ is desired only by agent $l_{i}^{j}$ and $x_{i}$, the two units of goods $l_{i}^{j}$ must be bought by them for the market clearance condition to hold as its price is non-zero. Therefore, $U\left(l_{i}^{j}\right)+$ $U\left(x_{i}\right)=2$.

Since the maximal utility of any trader is no more than 1 , we must have $U\left(l_{i}^{j}\right)=U\left(x_{i}\right)=1$. Notice that both trader $l_{i}^{0}$ and trader $l_{i}^{1}$ demand $\operatorname{good} x_{i}$ and the total amount of $\operatorname{good} x_{i}$ is 1 , we have $U\left(l_{i}^{0}\right)+U\left(l_{i}^{1}\right) \leq 1$.

Hence, for any $1 \leq i \leq n, U\left(l_{i}^{j_{i}}\right)=1$ and $U\left(l_{i}^{1-j_{i}}\right)=0$ for some $j_{i}=0,1$. The set of indices $\left\{j_{i} \mid 1 \leq i \leq n\right\}$ naturally yields a truth assignment for $\phi$ : $\left\{x_{i}=j_{i}, i=1,2, \ldots, n\right\}$.

For any clause $c_{k}$, assume w.o.l.g. $c_{k}=x_{i_{1}} \wedge$ $x_{i_{2}} \wedge \neg x_{i_{3}}$. Then

$U\left(l_{i_{1}}^{0}\right)+2 U\left(l_{i_{1}}^{1}\right)+U\left(l_{i_{2}}^{0}\right)+2 U\left(l_{i_{2}}^{1}\right)+2 U\left(l_{i_{3}}^{0}\right)+U\left(l_{i_{3}}^{1}\right) \leq 5$

The above inequality corresponding to the clause $c_{k}$ forces that in the truth assignment of $x_{i_{1}}, x_{i_{2}}$ and $x_{i_{3}}$ derived above, one of them will make the clause $c_{k}$ satisfied. Therefore the assignment derived from the market equilibrium makes $\phi$ satisfied.

Remark 1. The above economy always admits an equilibrium. Let $P(g)=1$ for any $g \in V \cup L$ and $P(g)=0$ for any $g \in C$. Then $U(b)=3 / 2$ for any $b \in V, U(b)=1 / 2$ for any $b \in L$ and $U(\phi)=0$. It is easy to check $(P, U)$ is an equilibrium.
An important property of the market $(A, E)$ is that for any equilibrium $(P, U)$ and all $i \in$ $\{1,2, \ldots, n\}$, either $U\left(x_{i}\right) \geq 3 / 2$, or $U\left(x_{i}\right)=$ $U\left(l_{i}^{j}\right)=1$ and $U\left(l_{i}^{1-j}\right)=0$ for some $j \in\{0,1\}$.

To see that, if both $P\left(l_{i}^{j}\right) \neq 0$ and $P\left(l_{i}^{1-j}\right) \neq 0$, then $U\left(l_{i}^{j}\right)+U\left(x_{i}\right)=2$ and $U\left(l_{i}^{1-j}\right)+U\left(x_{i}\right)=2$ as shown above. Therefore $U\left(l_{i}^{j}\right)=U\left(l_{i}^{1-j}\right)$. On the other hand, $U\left(l_{i}^{0}\right)+U\left(l_{i}^{1}\right) \leq 1$, also shown above. It follows that $U\left(x_{i}\right) \geq 3 / 2$ in this case.

Alternatively, assume that $P\left(l_{i}^{j}\right) \neq 0$ but $P\left(l_{i}^{1-j}\right)=0$. Then, $U\left(l_{i}^{j}\right)+U\left(x_{i}\right)=2$ by the former. Because of the latter, trader $b=l_{i}^{1-j}$ has a zero initial wealth and $U\left(l_{i}^{1-j}\right)=0$. Therefore the one unit of $x_{i}$ must be all sold to $b=l_{i}^{j}$ since it is desired only by $b=l_{i}^{j}$ and $b=l_{i}^{1-j}$. Therefore, $U\left(l_{i}^{j}\right)=1$. In this case, $U\left(x_{i}\right)=1$.

So we can add $n$ good to the market $(A, E)$, denoted by $\Sigma=\left\{\sigma_{1}, \sigma_{2}, \ldots, \sigma_{n}\right\}$. Let $E\left(\phi, \sigma_{i}\right)=5 / 4$ and $A\left(x_{i}, \sigma_{i}\right)=1$ for all $i$. The newly added goods $\sigma_{i}$ forces that $U\left(x_{i}\right) \leq 5 / 4$. Hence, in the modified economy $(A, E)$, there is a one-toone correspondence to its equilibria and satisfiable truth assignments of the Boolean formula $\phi$. This correspondence proves the \#P-complexity of counting the number of equilibria in Leontief economies:

Theorem 4. Counting the number of equilibria in Leontief economies is \#P-complete.

\subsection{Maximizing Individual Utility}

With similar technique in $\S 4.1$, we can show the NP-hardness of maximizing individual utility.

Theorem 5. For a trader in a Leontief economy and an arbitrary number $r$, it is NP-hard to decide whether there exists an equilibrium in which his utility is greater than $r$.

Proof. We extend the economy $M=(A, E)$ in the previous subsection. Add $n$ goods $\Theta=$ $\left\{\theta_{1}, \ldots, \theta_{n}\right\}$ and a trader $\psi$ to the market. We define $A$ and $E$ as follows:

$A(b, g)= \begin{cases}1, & \text { if } b=x_{i}, g=l_{i}^{j}, 1 \leq i \leq n, j=0,1 \\ 1, & \text { if } b=l_{i}^{j}, g=x_{i}, 1 \leq i \leq n, j=0,1 \\ 1, & \text { if } b=g=l_{i}^{j}, 1 \leq i \leq n, j=0,1 \\ 1, & \text { if } b=l_{i}^{j}, g=c_{k}, l_{i}^{j} \in c_{k}, \forall i, j, k \\ 2, & \text { if } b=l_{i}^{j}, g=c_{k}, l_{i}^{j} \notin c_{k} \\ & \text { and } l_{i}^{1-j} \in c_{k}, \forall i, j, k \\ 1, & \text { if } b=x_{i}, g=\theta_{i}, \forall i \\ 1, & \text { if } b=\psi, g \in \Theta \\ 0, & \text { otherwise. }\end{cases}$ 


$$
E(b, g)=\left\{\begin{array}{cl}
1, & \text { if } b=g=l_{i}^{j}, 1 \leq i \leq n, j=0,1 \\
1 / 2 n, & \text { if } b \in V, g \in V \cup L \\
1 / 2, & \text { if } b=\psi, g \in V \cup L \\
2, & \text { if } b=x_{i}, g=\theta_{i}, \forall i \\
5, & \text { if } b=\phi, g \in C \\
0, & \text { otherwise. }
\end{array}\right.
$$

We can declare that in any equilibrium $(P, U)$, $U(\psi) \geq 1$ if and only if $\max _{b \in V}\{U(b)\} \leq 1$. With similar argument in the proof of Theorem 3, there exists an equilibrium in which $\max _{b \in V}\{U(b)\} \leq 1$ if and only if the Boolean formula $\phi$ is satisfiable.

Remark 2. In the above economy, there always exists an equilibrium $(P, U)$ with the following values:

$$
\begin{gathered}
U(b)=\left\{\begin{array}{ccc}
3 / 2 \quad \text { when } \quad b \in V \\
1 / 2 \quad \text { when } \quad b \in L \text { or } b=\psi \\
0, & \text { otherwise. }
\end{array}\right. \\
P(g)=\left\{\begin{array}{ccc}
2, & \text { when } & g \in \Theta \\
1, & \text { when } & g \in V \cup L \\
0 & \text { otherwise. }
\end{array}\right.
\end{gathered}
$$

\section{Conclusions}

In this work, we prove that computing an equilibrium with optimal social/individual welfare in Leontief economies is NP-hard. In comparison, a major result of Codenotti, Saberi, Varadarajan and Ye [4] states that finding the equilibrium price is NP-hard. However, the economy they defined may not have an equilibrium price at all. The ultimate question remains open: what is the computational complexity for finding an equilibrium price, given that the economy admits one.

In addition, we develop a \#P-hard result for counting the number of equilibrium prices. Our \#P-hard result does not depend on the connection of market equilibrium to Nash equilibrium established by Codenotti, Saberi, Varadarajan and Ye [4]. The understanding of structural properties of Leontief economy is indeed very important in all the hardness proofs. We expect they would be helpful in moving forward our understanding of computational complexity of market equilibrium.

\section{References}

[1] K. J. Arrow and G. Debreu, Existence of an equilibrium for a competitive economy, Econometrica 22 (1954), no. 3, 265-290.
[2] B. Codenotti, B. McCune, and K. Varadarajan, Market equilibrium via the excess demand function, to appear in STOC'05.

[3] B. Codenotti, S. Pemmaraju, and K. Varadarajan, On the polynomial time computation of equilibria for certain exchange economies, to appear in SODA'05.

[4] B. Codenotti, A. Saberi, K. Varadarajan, and Yinyu Ye, Leontief economies encode nonzero sum two-player games, preliminary draft.

[5] B. Codenotti and K. Varadarajan, Efficient computation of equilibrium prices for markets with Leontief utilities, the proceedings of ICALP'04, pp. 371-382.

[6] V. Conitzer and T. Sandholm, Compleixity results about Nash equilibria, IJCAI'03, pp. 765771.

[7] X. Deng, C. Papadimitriou, and S. Safra, On the complexity of price equilibria, Journal of Computer and System Sciences 67 (2003), no. 2, 311-324.

[8] N. R. Devanur, The spending constraint model for market equilibrium: algorithmic, existence and uniqueness results, the proceedings of STOC'04, pp. 519-528.

[9] B. C. Eaves, Finite solution for pure trade markets with cobb-douglas utilities, Mathematical Programming Study (1985), no. 23, 226-239.

[10] R. Garg, S. Kapoor, and V. V. Vazirani, An auction-based market equilibrium algorithmfor the separable substitutibility case, the proceedings of APPROX'04, pp. 128-138.

[11] I. Gilboa and E. Zemel, Nash and correlated equilibria: Some complexity considerations, Games and Economic Behavior (1989), no. 1, 80-93.

[12] K. Jain, A polynomial time algorithm for computing the Arrow-Debreu market equilibrium for linear utilities, FOCS'04, pp. 286-294.

[13] E. Nenakhov and M. Primak, About one algorithm for finding the solution of the ArrowDebreu model, Kibernetica (1983), no. 3, 127128.

[14] Christos H. Papadimitriou, Algorithms, games, and the internet, Invited talk in STOC'01.

[15] H. Scarf, On the computation of equilibrium prices, in Ten Economic Studies in the Tradition of Irving Fisher, 1967.

[16] Yinyu Ye, A path to the Arrow-Debreu competitive market equilibrium, Available online http://www.stanford.edu/ yyye, 2004.

[17] — A note on exchange market equilibria with leontief's utility: Freedom of pricing leads to rationality, Available online http://www.stanford.edu/ yyye, 2005. 